\title{
Energy-Dependent Morphological Study of HESS J1857+026 with Fermi-LAT
}

\author{
Xiao-Lei Guo ${ }^{a, *}$ and Yu-Liang Xin ${ }^{a}$ \\ ${ }^{a}$ School of Physical Science and Technology, Southwest Jiaotong University, 610031 Chengdu, Sichuan, \\ China \\ E-mail: xlguo@swjtu.edu.cn, ylxin@swjtu.edu.cn
}

HESS J1857+026 is an extended gamma-ray source discovered by H.E.S.S., and considered to be a pulsar wind nebula candidate associated with PSR J1856+0245. Taking advantage of 11 years of Fermi-LAT data, we re-analyze the GeV gamma-ray emission from HESS J1857+026, and find that the $\mathrm{GeV}$ emission from this region is composed of two gamma-ray sources. The harder component Src T is coincident with HESS J1857+026, nevertheless the origin of the soft component Src A need further exploration. We present the results of energy-dependent extension analysis of Src T. HESS J1857+026 is a good target for LHAASO, CTA and HAWC. Future GeV and $\mathrm{TeV}$ observations will be helpful to investigate the particle transport mechanisms within this source.

$37^{\text {th }}$ International Cosmic Ray Conference (ICRC 2021)

July 12th-23rd, 2021

Online - Berlin, Germany

\footnotetext{
*Presenter
} 


\section{Introduction}

More than two hundred of very-high-energy (VHE; > $100 \mathrm{GeV}$ ) gamma-ray sources have been observed with the operations of ground-based Cherenkov telescopes, such as H.E.S.S. [1], MAGIC [2] and HAWC [3]. The identified VHE gamma-ray sources are associated with pulsar wind nebulae (PWNe), supernova remnants (SNRs), X-ray binaries an so on. The gamma-ray emission from the VHE gamma-ray sources could be produced by the inelastic proton-proton collisions process, in which $\pi^{0}$ decays into two gamma-ray photons subsequently, via the inverse Compton (IC) scattering process or through non-thermal Bremsstralung. Thus, these objects are tracers of relativistic particles, which are useful for understanding the diffusive shock acceleration (DSA) process.

HESS J1857+026 was first discovered as a VHE gamma-ray source without clear identified counterpart [4], and H.E.S.S. Collaboration [5] refined its extension. There is a pulsar PSR J1856+0245 in the direction of HESS J1857+026 [6] , which makes HESS J1857+026 become a potential PWN candidate. The spin period of PSR J1856+0245 is $81 \mathrm{~ms}$, the spin-down energy is $\dot{E}=4.6 \times 10^{36} \mathrm{erg} \mathrm{s}^{-1}$, the characteristic age $\tau_{c}$ is $21 \mathrm{kyr}$, and the distance is about $9 \mathrm{kpc}$ [6]. Subsequently, MAGIC carried out follow-up observations and presented energy-dependent morphology of this region [7]. They found two gamma-ray sources in this region for the data above $1 \mathrm{TeV}$, called MAGIC J1857.2+0263 and MAGIC J1857.6+0297. MAGIC J1857.2+0263 is considered to be a PWN powered by PSR J1856+0245, while MAGIC J1857.6+0297 may be associated with an HII region located at $3.7 \mathrm{kpc}$ and a possible gas cavity. In additon, 2HWC $\mathrm{J} 1857+027$, reported as $1 \mathrm{HWC} \mathrm{J} 1857+023$ previously, is considered to be associated with HESS $\mathrm{J} 1857+026$ [8].

The GeV emission from HESS J1857+026 was first detected with a point source hypothesis, and no gamma-ray pulsation of PSR J1856+0245 was observed [9]. And its GeV spectrum was described by a power law with a spectral index of $\Gamma=1.53 \pm 0.11_{\text {stat }} \pm 0.55_{\text {sys }}$.

Taking advantage of 11 years of Fermi-LAT data, we perform an energy-dependent analysis of HESS J1857+026, and constrain its spectrum with data in the energy range of $0.5-500 \mathrm{GeV}$.

\section{Fermi-LAT data and results}

We analyzed Fermi-LAT Pass 8 data collected from August 4, 2008 to August 4, 2019 with energies from $500 \mathrm{MeV}$ to $500 \mathrm{GeV}$ [10]. The region of interest (ROI) is a $10^{\circ} \times 10^{\circ}$ square centered at HESS J1857+026. To reduce the contamination from the Earth Limb, events with Earth zenith angle greater than $90^{\circ}$ were eliminated. Fermitools ${ }^{1}$ and the instrumental response function (IRF) of P8R3_SOURCE_V2 were used. The model contained all sources included in the Fermi-LAT fourth source catalog [4FGL; 11] within a radius of $15^{\circ}$ centered at HESS J1857+026, and the Galactic and isotropic gamma-ray backgrounds. During the analysis procedure, the binned maximum likelihood analysis method was applied.

\footnotetext{
${ }^{1}$ http://fermi.gsfc.nasa.gov/ssc/data/analysis/software/
} 


\subsection{Average Extension Analysis}

We performed the extension analysis in the energy ranges of $1-3 \mathrm{GeV}$ and $10-500 \mathrm{GeV}$. In the low energy band, only the events of "PSF3" type were selected to reduce the contamination from nearby sources. Figure 1 shows the TS maps of the two energy ranges after subtracting the sources included in the model (except for HESS J1857+026). In addition to 4FGL sources, there is still excess in the low energy, which is labeled as "Newptsrc". In the following analysis, we added "Newptsrc" in the model as a point source.

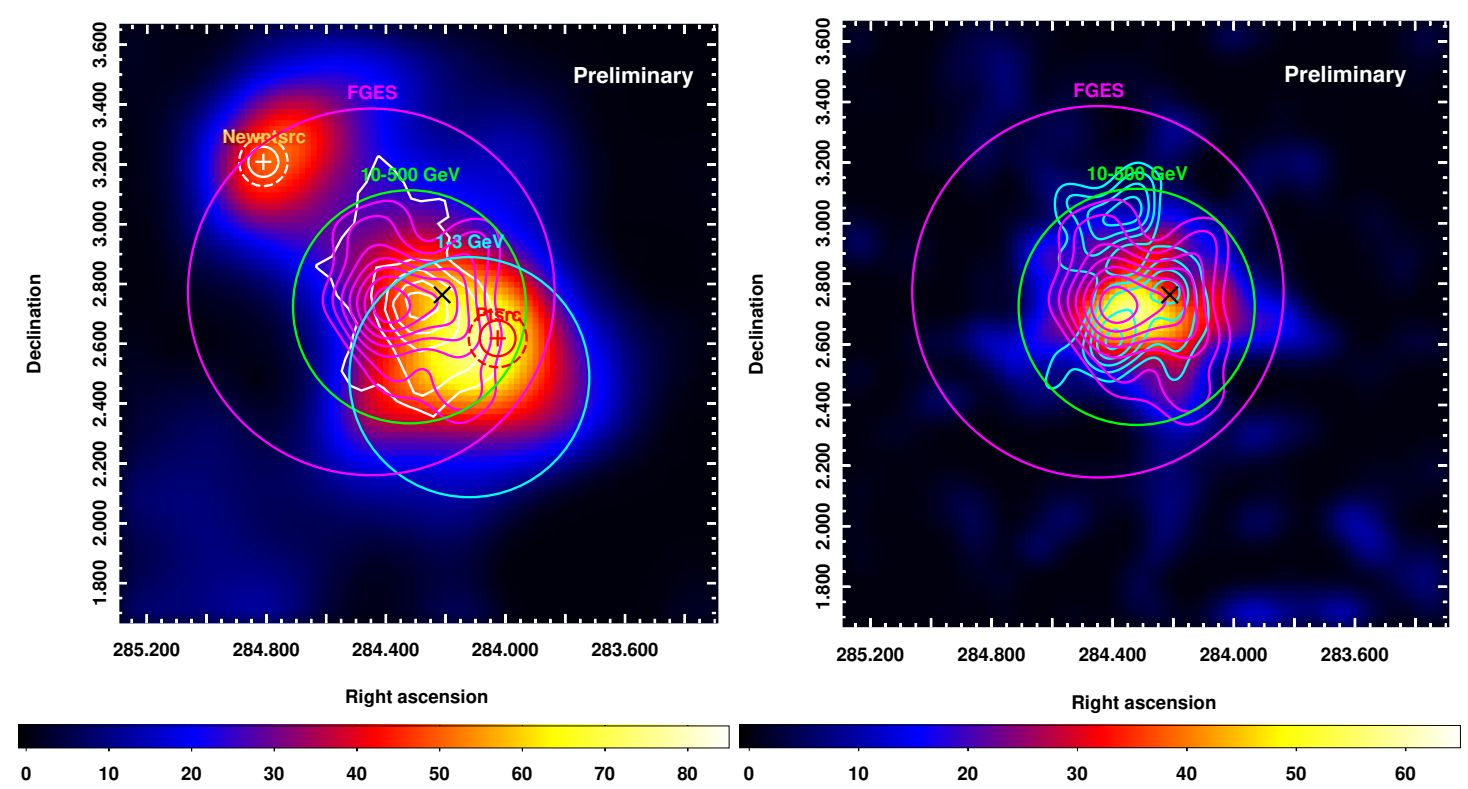

Figure 1: $2^{\circ} \times 2^{\circ}$ TS maps centered on HESS J1857+026. Left panel: TS map in the energy range of 1-3 GeV. The white and magenta contours indicate the H.E.S.S. image [5] and MAGIC observation below $1 \mathrm{TeV}$ [7], respectively. The white and red plus signs are the best-fit positions of "Newptsrc" and "Ptsrc", and their $1 \sigma$ and $2 \sigma$ error circles are marked by solid and dashed circles, respectively. The magenta circle is the spatial template of HESS J1857+026 given by FGES [12], and the cyan circle describes the $68 \%$ containment radius $\left(r_{68}\right)$ of the best-fit 2D Gaussian template with data below $3 \mathrm{GeV}$. And the black cross is the position of PSR J1856+0245. Right panel: TS map with data above $10 \mathrm{GeV}$. The cyan contours are the MAGIC image above $1 \mathrm{TeV}$ [7]. The green circle marks $r_{68}$ of 2D Gaussian model.

We noted that there is discrepancy between the GeV emission from HESS J1857+026 and the the spatial template given by Ackermann et al. [12]. Therefore, we refined the gamma-ray extensions in both low and high energy ranges with Fermipy ${ }^{2}$ package [13]. The point sourc marked as "Ptsrc", two-dimension (2D) Gaussian, uniform disk, as well as the H.E.S.S. image models were tested. Table 1 contains the results of extension measurements.

Both the 2D Gaussian and uniform disks templates can describe the $\mathrm{GeV}$ emission of both energy ranges. Above $10 \mathrm{GeV}$, the extension, we measured, is comparable to the H.E.S.S. observation [5]. The TS values of the $\mathrm{GeV}$ emission below $3 \mathrm{GeV}$ and above $10 \mathrm{GeV}$ (hereafter referred

${ }^{2}$ https://fermipy.readthedocs.io/en/latest/ 
to as "Src A" and "Src T" respectively) are 99 and 160, respectively. The photon index of Src A is $\Gamma=2.82 \pm 0.41$, which is much softer than that of $\operatorname{Src} \mathrm{T} \Gamma=1.85 \pm 0.10$, indicating different origin. Therefore, in the following analysis, both Src A and Src T are contained in the model with 2D Gaussian templates.

Table 1: Spatial Properties for the GeV Emission in the Direction of HESS J1857+026

\begin{tabular}{cccc}
\hline Energy Range & Spatial Template & $\begin{array}{c}r_{68} \\
(\mathrm{deg})\end{array}$ & $\begin{array}{c}\mathrm{TS}_{\mathrm{ext}} \\
(\mathrm{deg})\end{array}$ \\
\hline $1-3 \mathrm{GeV}$ & Point & - & 0 \\
& Uniform Disk (FGES) & 0.50 & -6 \\
& Uniform Disk & $0.39 \pm 0.04$ & 61 \\
& 2D Gaussian & $0.40 \pm 0.06$ & 61 \\
& H.E.S.S. Image & - & 22 \\
\hline $10-500 \mathrm{GeV}$ & Point & - & 0 \\
& Uniform Disk (FGES) & 0.50 & 69 \\
& Uniform Disk & $0.32 \pm 0.02$ & 104 \\
& 2D Gaussian & $0.39 \pm 0.04$ & 110 \\
& H.E.S.S. Image & - & 102 \\
\hline
\end{tabular}

\subsection{Spectral Analysis}

We performed the spectral analyses in the energy range of 0.5-500 GeV. The "PSF3" type events are selected for the data below $3 \mathrm{GeV}$. During the analysis process, the summed likelihood method was adopted. After fitting the data, the photon index of $\operatorname{Src} \mathrm{T}$ is $\Gamma=1.76 \pm 0.08$. The spectral index of Src A is $\Gamma=2.64 \pm 0.14$.

To study the spectral energy distributions (SEDs) of Src A and Src T, the data were divided into 9 logarithmically space energy bins. The SEDs are shown in Figure 2. The GeV spectrum of Src T connects with the TeV SED of HESS J1857+026 smoothly, suggesting that Src T is its $\mathrm{GeV}$ counterpart. From the SED, we can see that this source is a good target for the ground-based Cherenkov telescopes, such as LHAASO [15], CTA [16] and HAWC [3].

\subsection{Energy-Dependent Extension of Src T}

To further explore the energy-dependent behavior of $\mathrm{Src} \mathrm{T}$, we performed the analyses in the energy ranges of 5-15 GeV, $15-50 \mathrm{GeV}$ and $50-500 \mathrm{GeV}$. The extensions were measured by fitting 2D Gaussian templates with Fermipy. The results of the extension are listed in Table 2, and the TS maps are shown in Figure 3.

As shown in Table 2 and Figure 3, the extension of Src T increases with decreasing energy. A similar energy-dependent morphology was also observed in PWN HESS J1825-137 at TeV and $\mathrm{GeV}$ energies [17-19]. However, we notice that the variation of morphology is almost within $1 \sigma$ uncertainties. Since Src T is not bright enough to perform a more detailed morphology analysis, more Fermi-LAT and TeV observation data will be helpful to explore its morphology. 


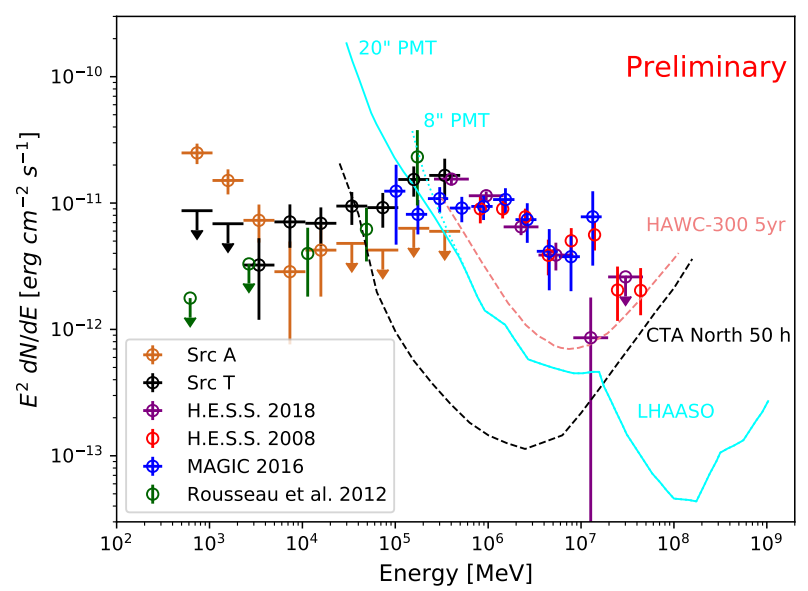

Figure 2: Gamma-ray SEDs of Src A and Src T. Fermi-LAT SED of Src A is marked by the chocolate circles, and the black circles represent the SED of Src $\mathrm{T}$ in this work. The green circles denote the GeV SED of HESS J1857+025 in previous work [9]. The red and purple circles indicate the H.E.S.S. observation by Aharonian et al. [4] and H.E.S.S. Collaboration [5], respectively. And the blue circles are the MAGIC observation in Aleksić et al. [7]. The cyan, black and pink lines represent the differential sensitivities of LHAASO [15], CTA [16] and HAWC [3], respectively.

Table 2: Extension Measurements of Src T in Different Energy Ranges

\begin{tabular}{ccc}
\hline Energy Range & $\begin{array}{c}r_{68} \\
(\mathrm{deg})\end{array}$ & TS Value \\
\hline $5-15 \mathrm{GeV}$ & $0.57 \pm 0.11$ & 62 \\
$15-50 \mathrm{GeV}$ & $0.49 \pm 0.11$ & 48 \\
$50-500 \mathrm{GeV}$ & $0.31 \pm 0.04$ & 69 \\
\hline
\end{tabular}

\section{Conclusion}

Using 11 years of Fermi-LAT observations, we performed the energy-dependent analysis of $\mathrm{GeV}$ emission in the direction of HESS J1857+026. We found that the $\mathrm{GeV}$ emission from HESS $\mathrm{J} 1857+026$ is composed of two gamma-ray sources called Src A and Src T. The GeV emission from Src T is spatially coincident with HESS J1857+026 and its GeV spectrum connects with the TeV SED of HESS J1857+026 smoothly, indicating same physical origin. But the nature of Src A is not clear. In additon, we report, for the first time, the continue morphology changes of $\mathrm{GeV}$ emission from HESS J1857+026. However, due to the limited statistics, the uncertainties of the extension variation is relatively large.

HESS J1857+026 is one of the peculiar gamma-ray sources, which show energy-dependent morphology. This source is a good target for LHASSO [15], CTA [16] and HAWC [3]. 


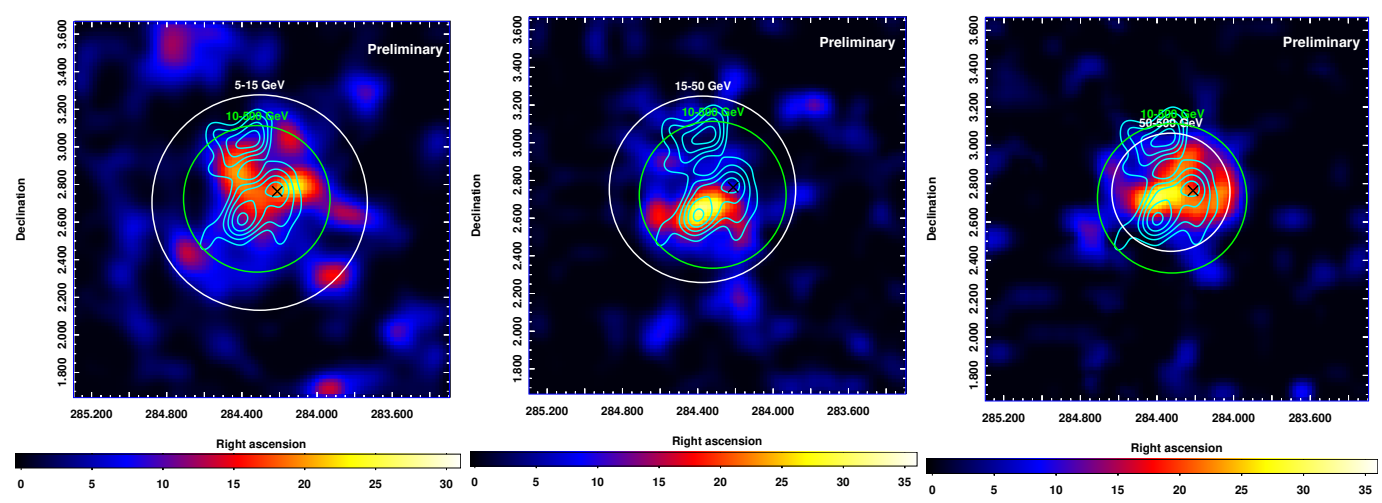

Figure 3: $2^{\circ} \times 2^{\circ}$ TS maps centered on HESS J1857+026 for the energy ranges of 5-15 GeV (left), 15-50 $\mathrm{GeV}$ (middle) and 50-500 GeV (right). The green circles indicate the $r_{68}$ of 2D Gaussian template with data above $10 \mathrm{GeV}$, and the white circles mark the $r_{68}$ for different energy bands.

\section{Acknowledgements}

This work is supported by the Fundamental Research Funds for the Central Universities (No. 2682021CX074, No. 2682021CX073), and the Natural Science Foundation for Young Scholars of Jiangsu Province, China (No. BK20191109).

\section{References}

[1] Aharonian, F., Akhperjanian, A. G., Aye, K.-M., et al. 2004, Astropart. Phys., 22, 109

[2] Aleksić, J., Ansoldi, S., Antonelli, L. A., et al. 2016, Astropart. Phys., 72, 61

[3] Abeysekara, A. U., Alfaro, R., Alvarez, C., et al. 2013, Astropart. Phys., 50, 26

[4] Aharonian, F., Akhperjanian, A. G., Barres de Almeida, U., et al. 2008, A\&A, 477, 353

[5] H.E.S.S. Collaboration 2018, A\&A, 612, A1

[6] Hessels, J. W., Nice D. J., Gaensler B. M., et al. 2008, ApJL, 682, L41

[7] Aleksić, J., Ansoldi, S., Antonelli, L. A., et al. 2014, A\&A, 571, A96

[8] Abeysekara, A. U., Albert, A., Alfaro, R., et al. 2017, ApJ, 843, 40

[9] Rousseau, R., Grondin, M.-H., Van Etten, A., et al. 2012, A\&A, 544, A3

[10] Atwood, W. B., Abdo, A. A., Ackermann, M., et al. 2009, ApJ, 697, 1071

[11] Abdollahi, S., Acero, F., Ackermann, M., et al. 2020, ApJS, 247, 33

[12] Ackermann, M., Ajello, M., Baldini, L., et al. 2017, ApJ, 843, 139 
[13] Wood M., Caputo R., Charles E. et al., 2017, ArXiv:1707.09551

[14] Lande, J., Ackermann, M., Allafort, A., et al. 2012, ApJ, 756, 5

[15] Bai, X., Bi, B. Y., Bi, X. J., et al. 2019, ArXiv:1905.02773

[16] Cherenkov Telescope Array Consortium, Acharya, B. S., Agudo, I., et al. 2019, Science with the Cherenkov Telescope Array (Singapore: World Scientific )

[17] Aharonian, F., Akhperjanian, A. G., Bazer-Bachi, A. R., et al. 2006, A\&A, 460, 365

[18] H.E.S.S. Collaboration 2019, A\&A, 621, A116

[19] Principe, G., Mitchell, A. M. W., Caroff, S., et al. 2020, A\&A, 640, A76 